\title{
Les protéines de choc thermique chez Lactococcus lactis : synthèse et régulation ; thermotolérance
}

\author{
P Boutibonnes
}

\author{
Laboratoire de microbiologie de l'environnement, \\ IRBA, Université de Caen, 14032 Caen cedex, France
}

\begin{abstract}
Résumé - On retrouve chez Lactococcus lactis les deux composantes habituelles de la réponse au choc thermique : la synthèse d'un petit nombre de protéines spécifiques dites hsps (pour "heat shock proteins") et l'élévation phénotypique de la résistance naturelle à la chaleur dite thermotolérance. Le passage brutal de 30 à $40^{\circ} \mathrm{C}$ s'accompagne de l'amplification de la synthèse de 13 à 16 protéines parmi lesquelles sont reconnues par immunodétection sept protéines homologues des hsps d'Escherichia coli (ClpB, DnaJ, Dnak, GroEL,GrpE, Lon) et de Bacillus subtilis $\left(\sigma^{43}\right)$. Six des gènes codant pour ces polypeptides-clés, surexprimés après l'élévation thermique, ont été clonés et séquencés ; trois d'entre eux ont été localisés sur le chromosome ; ils montrent d'assez faibles différences avec leurs modèles bactériens déjà identifiés. La régulation de leur expression semble assez proche de celle des gènes thermorégulés de $B$ subtilis. La thermotolérance s'exprime dans des conditions voisines; elle est maximale à $42^{\circ} \mathrm{C}$ lorsque la température d'épreuve est de $52^{\circ} \mathrm{C}$. Toutefois les deux phénomènes ne sont pas induits par les mêmes agents "stressants" thermomimétiques. Comme chez les autres organismes ou cellules (eucaryotes et procaryotes), il est difficile de lier par un strict principe de causalité l'apparition de ce petit jeu de protéines à l'exhaussement de la résistance à la chaleur, déclenchés tous deux par des températures infra-létales.
\end{abstract}

\section{Lactococcus lactis / protéine de choc thermique / thermotolérance}

Summary - Heat-shock proteins in Lactococcus lactis, synthesis and regulation; thermotolerance. Like in other organisms studied today, the heat shock response in Lactococcus lactis is characterized by the synthesis of a set of specific proteins, the so-called heat shock proteins or hsps and by the elevation of natural thermic resistance. When L lactis cells are heat-shocked from $30^{\circ} \mathrm{C}$ to $40^{\circ} \mathrm{C}$ they induce 13 to $16 \mathrm{hsps}$. Among these, seven are characterized and were found to be immunologically related to the Escherichia coli ClpB, DnaJ, DnaK, GroEL, GrpE, Lon and to the Bacillus subtilis major sigma factor $\left(\sigma^{43}\right)$. Six of these $h$ s genes were cloned and sequenced and show a high degree of homology with other bacterial hs genes. Three of them were located on the genetic map of the chromosome. Thermotolerance was expressed in the same conditions. Yet, this phenomenon was maximal at $42^{\circ} \mathrm{C}$ when the challenge lethal temperature was $52^{\circ} \mathrm{C}$. Like in other organisms or eukaryotic and prokaryotic cells it is difficult to correlate, in L lactis, the elevation of transient induced tolerance to the amplification of one or some $h s p(s)$ triggered by intermediate non-lethal temperatures.

\section{Lactococcus lactis / heat shock protein / thermotolerance}




\section{INTRODUCTION}

Il y a une trentaine d'années, Ritossa (1962) rapportait, dans une courte note, un étrange phénomène qu'il avait observé dans un élevage de drosophiles, soustrait à son environnement thermique habituel $\left(25^{\circ} \mathrm{C}\right):$ : Si les larves écrivait-il, sont soumises à un choc thermique à $30^{\circ} \mathrm{C}$ pendant 30 minutes environ, il s'ensuit un brutal changement du profil des "puffs" ou boursouflures des chromosomes". Ces "gonflements" qui intéressent toujours les mêmes régions des chromosomes polytènes des glandes salivaires des diptères traduisent une intense activité transcriptionnelle : "Les puffs sont des gènes actif́s" ajoutait Ritossa. L'apparition de ces hernies, ou nodules, est suivie, quelques minutes après par la synthèse de protéines inhabituelles (Tissières et al, 1974), qui furent appelées hsps (heat shock proteins). Cette première observation ouvrit des perspectives fertiles ; le même phénomène, marqué par un bouleversement du spectre protéique, consécutif à une augmentation de température, fut reconnu chez de très nombreuses espèces animales ou végétales avant d'être identifié chez Escherichia coli (Lemaux et al, 1978 ; Yamamori et al, 1978). La réponse à ce "choc" thermique, comme toutes celles déclenchées par d'autres stress physico-chimiques, permet aux organismes de mettre en place des dispositifs physiologiques temporaires qui assurent un ajustement des voies métaboliques aux nouvelles contraintes ou menaces de l'environnement. Ces réponses sont bénéfiques en ceci qu'elles confèrent aux organismes la capacité de survivre, du moins transitoirement, à des conditions ou épreuves létales, homologues ou proches de celles qui en ont commandé le déclenchement. On nommera tolérance cette amélioration phénotypique de la survie et, plus précisément thermotolérance, l'exhaussement de la résistance "naturelle» de l'organisme à la chaleur. Les deux phéno- mènes, synthèse d'hsps d'une part, thermotolérance d'autre part, coexistent. Sont-ils pour autant liés par un principe de causalité (Boutibonnes et al, 1992) : la question est toujours irrésolue...

Quelle que soit l'assise biochimique de la tolérance, la réponse bipartite à médiation biochimique et physiologique est universelle: présente chez tous les êtres vivants, chez toutes les cellules en culture, qu'elles soient embryonnaires, adultes ou néoplasiques, elle se déroule selon les mêmes modalités qui font intervenir successivement : un "stresseur" ou stimulateur, la chaleur bien sûr, mais aussi tout autre agent dit thermomimétique qui, comme l'alcool, produit un déclenchement analogue ; un capteur ou "senseur" qui est une cible cellulaire sensible et qui dans le cas présent est une (ou des) protéine (s) ; un signal de nature inconnue, sinon à considérer que la protéine altérée assure elle-même cette fonction ; un effecteur responsable de la bascule transcriptionnelle qui conduit à la "différenciation métabolique" ; des modulateurs ou régulateurs négatifs qui tempèrent l'amplitude de la réponse.

Universelle, la réponse est transitoire : elle est manifeste quelques minutes après le choc mais elle est de courte durée. En général 1 ou $2 \mathrm{~h}$ après le déclenchement du dispositif, les organismes ou les cellules retournent à un état de repos qui est une nécessité, pour obéir à une nouvelle sollicitation du milieu extérieur.

Universelle et transitoire, la réponse qui s'est sans doute mise en place précocement au cours de l'évolution, est conservée dans tout le monde vivant : on retrouve, même si elles portent des noms différents les mêmes protéines présentant, au moins dans leurs zones fonctionnelles, les mêmes structures, et assurant les mêmes fonctions ; aussi en est-il de la hsp 60 , de la cpn 60 des cellules eucaryotes et de GroEL d'E coli. Elles possèdent toutes trois la propriété de veiller au repliememt orthodoxe des pro- 
téines immatures, ou de restaurer la conformation native de leurs structures anormales irrégulières ou agrégées, provoquées par des agents chaotropiques.

Parce qu'elle est universelle et conservée, parce qu'elle est la plus anciennement connue, parce qu'elle permet aux organismes de s'affranchir de l'impérieux pouvoir du réel, la réponse au choc thermique peutêtre considérée comme l'exemple des stratégies d'adaptation ou d'ajustement (coping) à l'environnement. Elle représente aussi un modèle simple et efficace de différenciation programmatique : son recours puis sa mise en œuvre révèlent, au sein du génome, l'existence de projets enfouis, occasionnellement réactivés. En cela, elle ne cesse de nous fasciner...

II semble inévitable, lorsqu'on aborde chez les bactéries la physiologie ou la régulation de la réponse au choc thermique, de se référer, à ce qui, aujourd'hui, fait figure de système codifié, voire de paradigme : le modèle $E$ coli ; non seulement parce qu'il est accompli (un deuxième régulon intriqué au système multigénique classique et le complétant vient d'être individualisé (Rouvière et al, 1995 ; Raina et al, 1995)) mais aussi à cause de sa "beauté" et, de son évidente simplicité : ainsi la bascule métabolique se noue autour d'un relais de facteurs sigma. Nous y ferons bien sûr référence.

Cependant Lactococcus lactis se distingue de l'archétype bactérien par quatre traits singuliers : c'est une bactérie Gram positive, mésophile, microaérophile qui a emprunté des voies évolutives distinctes de celle des protéobactéries ; son GC \% est bas, 34 à $38 \%$ (Sandine et al, 1972) contre 48 à $52 \%$ pour E coli (Brenner, 1984); son génome est de petite taille: de 2 à $2,7 \mathrm{MB}$ (Le Bourgeois et al, 1989 ; Tanskanen et al, 1990), alors qu'il est de 4,2 à 4,5 MB chez $E$ coli. Enfin sa niche écologique, le lait, les produits laitiers, est éloignée de celle des bactéries entériques (Sandine et al,
1972) ; L lactis y est soumis, au cours des processus de biotransformation auxquels il participe à de nombreuses sautes thermiques. Ces constatations laissent supposer que la réponse, même si elle est phylogéniquement conservée est, chez cette espèce, assez divergente du modèle conventionnel accepté. Évoquer la réponse au choc thermique, et plus particulièrement les hsps qui en sont la manifestation la plus flagrante, c'est décrire: (1) les circonstances de leur apparition, leurs nombre et qualités ; (2) le mode de régulation de leur synthèse ; (3) leur participation hypothétique au développement de la thermotolérance.

\section{LES PROTÉINES DE CHOC THERMIQUE}

Lorsqu'une culture de $L$ lactis, incubée à 30 ou $35^{\circ} \mathrm{C}$ est soudainement exposée à une température plus élevée ( 40 à $42^{\circ} \mathrm{C}$ ), le profil protéique des cellules qui la compose, analysé par électrophorèse bidimensionnelle après marquage à la ( $\left.{ }^{35} \mathrm{~S}\right)$ méthionine, révèle la disparition d'un grand nombre de protéines, et l'amplification, par un facteur de 2 à 3 , de la synthèse de 13 (Whitaker et Batt, 1991) ou 16 (Boutibonnes et al, 1991 ; Auffray et al, 1992) polypeptides (fig 1). Leur masse moléculaire (MM) s'étage de 20 à 100 kDa. Par immunodétection, sont identifiées 5 protéines interagissant avec des anticorps dirigés contre les hsps majeures d' E coli: DnaK, DnaJ, GroEL, GrpE (Auffray et al, 1992), et Lon (résultat non publié) ; deux autres protéines réagisserit avec les anticorps du facteur sigma majeur $\left(\sigma^{43}\right)$ de Bacillus subtilis (Auffray et al, 1992), et de la hsp 104 de Saccharomyces cerevisiae, (homologue de la protéase ClpB d'E coli) (Boutibonnes et al, 1995). Bien qu'elles soient immunologiquement semblables à leurs homologues bactériens connus, avec lesquels elles présentent des épitopes communs, elles s'en éloignent quelquefois par leur masse moléculaire (tableau I) : c'est le 

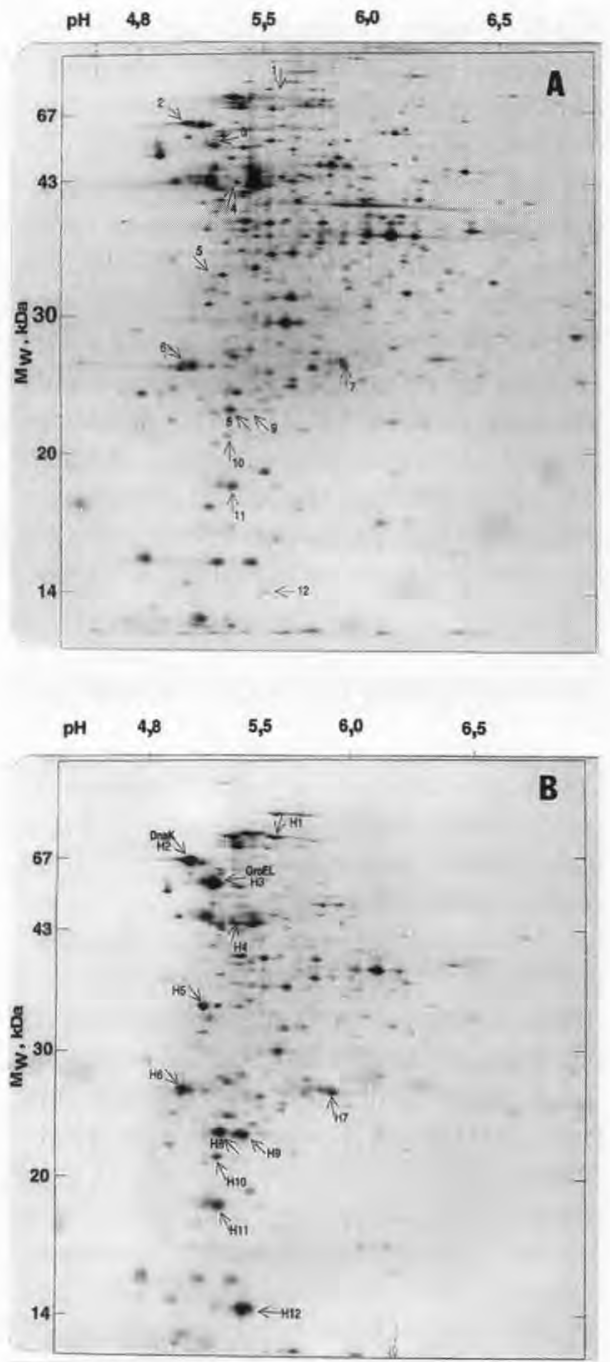

Fig 1. Électrophorèse bidimensionnelle en gel de polyacrylamide d'un extrait cellulaire d'une culture de Lactococcus lactis IL 1403 à $30^{\circ} \mathrm{C}$ (A) ou à $42{ }^{\circ} \mathrm{C}(\mathrm{B})$. Les protéines de choc thermique (hsps) sont désignées par la lettre $\mathrm{H}$ et par un chiffre. Deux d'entre elles, DnaK et GroEL on été identifiées à l'aide d'anticorps dirigés contre des hsps d'Escherichia coli.

Bidimensional electrophoresis analysis of a cellular extract of growing cells of Lactococcus lactis IL 1403 at $30^{\circ} \mathrm{C}(A)$ or at $42^{\circ} \mathrm{C}(B)$. Heat shock proteins (hsps) are designated by the letter $\mathrm{H}$ and by a number. Two of these, DnaK and GroEL were identified with antisera raised against hsps of Escherichia coli. cas de la protéase Lon ( $55 \mathrm{kDa}$ contre 94 ou 87 chez E coli), de GrpE ( 94 kDa alors qu'elle est chez $E$ coli classée parmi les hsps à faible $\mathrm{MM}$ ) et du facteur $\sigma^{43}$ équivalent (ce dernier, estimé à $51-55 \mathrm{kDa}$, riche en résidus chargés ne migre pas sur les gels de polyacrylamide au même niveau que les molécules de référence) (Burton et al, 1981). Sa masse moléculaire réelle, inférée de la séquence génique, est de $39 \mathrm{kDa}$ (Gansel et al, 1993 a, b).

Bien qu'elles ne représentent que le tiers des hsps repérées, les protéines identifiées appartiennent à tous les grands groupes (Georgopoulos et al, 1990 ; Morimoto et al, 1994). Ce sont des protéases endocellulaires (ClpB et Lon); des composantes de la machinerie de chaperonnage : chargées d'assurer le repliement régulier des oligopeptides, elles participent par un mécanisme ATP-dépendant à la désagrégation et à la restauration des protéines fonctionnelles endommagées par la chaleur (Dnak et GroEL) (Craig et al, 1993) ; des protéines d'assistance des chaperons moléculaires (partner proteins) (GrpE et DnaJ). Une autre protéine occupe dans la régulation de la réponse au choc thermique une position clé, c'est le facteur $\sigma^{39}$. Chaperons moléculaires et protéases endocellulaires appartiennent à des systèmes convergents dont l'activité est strictement coordonnée, au point d'ailleurs que l'une des protéases du groupe de hsps 100, Clp X a, chez E coli une activité de chaperonnage (Wawrzynow et al, 1995).

Un certain nombre d'agents chimiques, dits "thermomimétiques" (Boutibonnes et al, 1995), dont l'éthanol (à 2 ou $4 \%$ ) est le représentant le plus exemplaire, peuvent à température ordinaire, induire la synthèse de ces protéines singulières chez les procaryotes. Ces inducteurs ou stresseurs peuvent être répartis en quatre groupes (tableau II) :

- les analogues d'acides aminés, telles l'azétidine analogue de la proline et la cana- 
Tableau I. Protéines de choc thermique de Lactococcus lactis ( $L$ lactis subsp lactis IL 1403 et $L$ lactis NCDO 763) identifiées par immunodétection (Western-Blotting) à l'aide d'anticorps dirigés contre des hsps d'Escherichia coli, de Bacillus subtilis et de Saccharomyces cerevisiae.

Heat shock proteins of Lactococcus lactis (L lactis subsp lactis IL 1403 and L lactis NCDO 763) characterized by immunodetection (Western-Blotting) with antisera raised against heat shock proteins of Escherichia coli, Bacillus subtilis and Saccharomyces cerevisiae.

\begin{tabular}{|c|c|c|c|}
\hline Groupe (a) & $\begin{array}{l}\text { hsps de référence: } \\
\text { origine et nom }\end{array}$ & $M M(k D a)(b)$ & $\begin{array}{c}M M(k D a) \text { des hor } \\
\text { de L lactis }\end{array}$ \\
\hline hsp 100 & $\begin{array}{c}\text { E coli } \\
\text { ClpB (d); S cerevisiae } \\
\text { hsp } 104 \text { (d) }\end{array}$ & $93(+79)$ & 94 \\
\hline hsp 90 & $\begin{array}{c}\text { E coli } \\
\text { Lon (La) }\end{array}$ & 94 ou 87 & 55 \\
\hline hsp70 & $\begin{array}{c}E \text { coli } \\
\text { DnaK }(e)^{\star}\end{array}$ & 69 & 70 \\
\hline hsp60 & $\begin{array}{c}\text { E coli } \\
\text { GroEL * }\end{array}$ & 63 & 63 \\
\hline \multirow[t]{2}{*}{ hsp $27 / 28$} & $\begin{array}{l}\text { E coli } \\
\text { DnaJ * } \\
\text { GrpE * }\end{array}$ & $\begin{array}{l}41 \\
22\end{array}$ & $\begin{array}{c}43(+94) \\
94\end{array}$ \\
\hline & $\begin{array}{c}\text { B subtilis } \\
\text { Sigma majeur }{ }^{*}\left(\sigma^{43}\right)\end{array}$ & 43 & $51-55$ (f) \\
\hline
\end{tabular}

(a) D'après Morimoto et al, 1994 ; (b) D'après Georgopoulos et al, 1990 ; (c) D'après Whitaker et Batt, 1991 ; Auffray et al, 1992 ; Boutibonnes et al, 1995 ; (d) ClpB est l'homologue bactérien de la hsp 104 de Saccharomyces cerevisiae (Sanchez et Lindquist, 1990) ; l'anticorps utilisé était dirigé contre la protéine de la levure : (e) L'astérisque désigne les protéines de $L$ lactis dont le gène a été cloné et séquencé ; (f) En réalité 39 , calculé d'après la composition en acides aminés déduite de la séquence nucléotidique.

(a) According to Morimoto et al, 1994; (b) According to Georgopoulos et al, 1990; (c) According to Whitaker and Batt, 1991; Auffray et al, 1992; Boutibonnes et al, 1995; (d) ClpB is the bacterial homolog of Sacharomyces cerevisiae hsp104; antiserum used was raised against the yeast hsp104 (Sanchez and Lindquist, 1990); (e) Asterisk indicates proteins whose gene was cloned and sequenced; ( $f$ ) Real MW of 39 calculated after aminoacid composition deduced from nucleotidic sequence.

vanine, proche de l'arginine, qui provoquent, par défaut de repliement conforme, la formation des protéines irrégulières ;

- la puromycine, qui interrompt l'édification de la chaîne polypeptidique et forme des protéines incomplètes (peptidyl-puromycine) ;

- les antibiotiques, qui agissent sur l'étape traductionnelle de la synthèse protéique et entraînent, par erreur de lecture ou défaut d'élongation, la production des protéines incorrectes ou tronquées ;

- les agents chaotropiques, qui affectent le repliement régulier des protéines ordinaires ou occasionnent la formation d'agrégats ; certains d'entre eux, la caféine, la coumarine, l'acétylsalicylate, le chlorure de baryum sont réputés être, chez les cellules euca- 
Tableau II. Effet de la chaleur et de divers agents "thermomimétiques" sur la néosynthèse de protéines de choc thermique (hsps) et sur le développement de la thermotolérance chez Lactococcus lactis ( $L$ lactis subsp lactis IL 1403 et L lactis NCDO 763).

Effect of heat and some "thermomimetic" agents on the de novo synthesis of heat shock proteins and on the development of thermotolerance in Lactococcus lactis $\mathrm{L}$ lactis subsp lactis $\mathrm{L} 1403$ and L lactis NCDO 763).

Agents (a)

Amplification (b)

$F T(c)$

DnaJ Grpe GroEL hsp 104

Température $42^{\circ} \mathrm{C}$

Alcool [2 ou $4 \%(\mathrm{vol} / \mathrm{vol})]$

Puromycine

Streptomycine

Kanamycine

Erythromycine

Spiramycine

Chloramphénicol

Azétidine

Canavanine

Chlorure de cadmium (e)

Chlorure mercurique

Azide de sodium

$\beta$-mercapto éthanol

Phase stationnaire

$\begin{array}{cc}++ & ++ \\ + & + \\ - & ++ \\ + & ++ \\ +- & - \\ + & 0 \\ + & 0 \\ - & 0 \\ - & 0 \\ - & 0 \\ 0 & 0 \\ 0 & 0 \\ 0 & 0 \\ 0 & 0 \\ - & -\end{array}$

+++
$+1-$
0
0
0
0
0
0
0
0
++
-
-
++
-

(a) Tous les agents chimiques sont testés à trois concentrations correspondant aux CMI (Concentration minimale inhibitrice) 20,50 et $80 \%$; (b) -, pas d'amplification; + , amplification visible ; +++ , amplification $\geq 3 ; 0$, recherche omise ; (c) FT, facteur de thermotolérance (voir texte) est noté :,$- \leq 1 ;+$, de 3 à $10 ;++$, de 10 à $100 ;+++, \geq$ à 100 ; (d) ++ avec L lactis ML3 ; sans effet sur L lactis 1403. Pour les 2 souches l'acquisition d'une alcoolotolérance [ethanol à $20 \%$, (vol/vol)] se fait à un niveau élevé ; (e) seuls figurent dans le groupe des composés chimiques les 4 substances, parmi les 16 testées, responsables d'une réponse positive. Sont sans effet sur le développement de la thermotolérance les composés suivants : méthanol, chlorure de baryum, sulfate de cuivre, chlorure de zinc, acétate de thallium, arsenite de sodium, tellurite de potassium, caféine, coumarine, acétylsalicylate, diméthyl sulfoxide (DMSO) et bleu de méthylène.

(a) The chemical agents were tested at 3 concentrations corresponding to the IMC (inhibitory minimal concentration) 20,50 and $80 \%$; (b) -, no amplification; +, obvious amplification; +++, amplification $\geq 3 ; 0$, not determined; (c) FT, thermotolerance factor (see text) was noted: $-\leq 1 ;+$, from 3 to $10 ;++$, from 10 to $100 ;+++, \geq 100 ;$ (d) ++ with L lactis ML3; without effect with L lactis IL 1403. These two strains showed acquisition of alcohol-tolerance (20\% ethanol, (vol/vol)) at high levels; (e) Four chemicals only, among the 16 tested, gave a positive response. Are without effect the following chemicals: methanol, baryum chloride, copper sulfate, zinc chloride, thallium acetate, sodium arsenite, potassium tellurite, caffeine, coumarin, acetyl salicylate, dimethyl sulfoxide (DMSO) and methylene blue.

ryotes en culture des inducteurs efficaces d'hsps. À l'exception de la chaleur ellemême, et à un moindre degré, de l'alcool puis de la puromycine et des antibiotiques, la plupart des inducteurs froids de la synthèse des hsps, reconnus dans la littéra- ture, sont sans effet chez L lactis (tableau II). À l'inverse, la majorité d'entre eux déclenchent un processus physiologique, indépendant de l'amplification de quelques protéines privilégiées, qui conduit à l'acquisition de la thermotolérance. De plus, si l'entrée 
des cellules en phase stationnaire détermine chez $E$ coli la mise en place d'un système multigénique complexe, le stimulon de phase stationnaire, qui intègre quelques éléments du régulon de choc thermique, ce qui se traduit par l'amplification de GroEL et de DnaK, il n'en est rien chez $L$ lactis alors que, dans cette phase terminale de la croissance, les niveaux de résistance à de nombreuses épreuves létales y compris la chaleur, sont élevées (Hartke et al, 1994).

\section{RÉGULATION DE LA SYNTHÈSE DES PROTÉINES DE CHOC THERMIQUE}

Par quelle voie, et selon quel mode de régulation s'effectue la synthèse des hsps? Construire un modèle pertinent, c'est en premier lieu identifier les structures géniques qui en commandent la formation pour, ensuite, se référer aux schémas bactériens établis : en particulier, à celui d' $E$ coli élevé au rang de modèle canonique mais aussi à celui moins élaboré, peut-être aussi plus archaïque de Bacillus subtilis (Arnosti et al, 1986). Six gènes des hsps majeures de $L$ lactis ont été jusqu'à ce jour reconnus, clonés puis séquencés. Un septième gène thermorégulé, homologue de $f t s H$ (hflB) d' $E$ coli vient d'être identifié (Nilsson et al, 1994) ; son produit, la protéine transmembranaire $\mathrm{HflB}$, douée d'une faible activité ATPasique participerait comme DnaK, DnaJ, GrpE - à la régulation négative de la réponse subordonnée à $\sigma^{32}$ dont il contrôlerait la dégradation et par là, le niveau endocellulaire (Herman et al, 1995 ; Duwat et al, 1995). A cet ensemble non clos, il faut ajouter le gène recA, pièce centrale du régulon SOS : son produit, la protéine Rec $A$, exercerait un effet régulateur sur le système multigénique de choc thermique en supervisant le taux de HflB (Duwat et al, 1995).

Les caractéristiques essentielles de ces gènes figurent dans les tableaux III et IV ; nous résumerons, ci-dessous, leurs particularités les plus saillantes.

Les gènes sont isolés ou groupés en petits opérons, qui rappellent ceux d' $E$ coli (groEL-ES), (Kim et Batt, 1993), de B subtilis (grpE-dnaK) (Barril et al, 1994), ou des deux espèces (rpoD-dnaE) ( $B$ subtilis) ou (rpoD - dnaG) ( $E$ coli). Cependant, chez $L$ lactis l'opéron dnaK comprend deux autres cadres ouverts de lecture (orf) (Eaton et al, 1993), et dnaJ, habituellement couplé à dnaK fait défaut (Van Asseldonk et al, 1993). De la structure opéronique rpoD-dnaE, constituant l'opéron MMS (macro molecular synthesis) est absent, en amont, le gène $r p s U$ dont le produit est impliqué dans la traduction (Araya et al, 1993).

L'alignement des séquences en acides aminés des produits des gènes clonés, déduites des séquences nucléotidiques, avec celles des protéines homologues de diverses espèces bactériennes ( $E$ coli, $B$ subtilis, $B$ megaterium, Staphylococcus aureus), démontre une homologie importante (tableau IV). Cependant, chez $L$ lactis comme chez les autres bactéries Grampositives on note la délétion de certaines parties : ainsi la région V2 du facteur sigma majeur d'E coli (rpoD) (Eaton et al, 1993) qui compte chez les bactéries Gram-négatives 250 acides aminés est réduite ici à quelques résidus (Araya et al, 1993 ; Gansel et al, 1993b) ; de même, la protéine DnaK, est amputée dans sa partie $\mathrm{N}$ terminale d'une séquence de 24 acides aminés ce qui justifie une masse moléculaire calculée plus faible que celle de la molécule homologue d'E coli (Eaton et al, 1993).

Les cadres ouverts de lecture ou les opérons de $L$ lactis sont précédés :

- par un promoteur végétatif présentant des séquences -35 et -10 caractéristiques (TTGACA et TAAAAT) (Eaton et al, 1993) ; - par une IR, (répétition inversée) de 9 à $12 \mathrm{pb}$ formant une structure en tige-etboucle (stem-loop), identique à celle repérée 
Tableau III. Principales caractéristiques des gènes de choc thermique clonés et séquencés chez diverses souches de Lactococcus lactis. Major characteristics of cloned and sequenced heat shock genes of some strains of Lactococcus lactis.

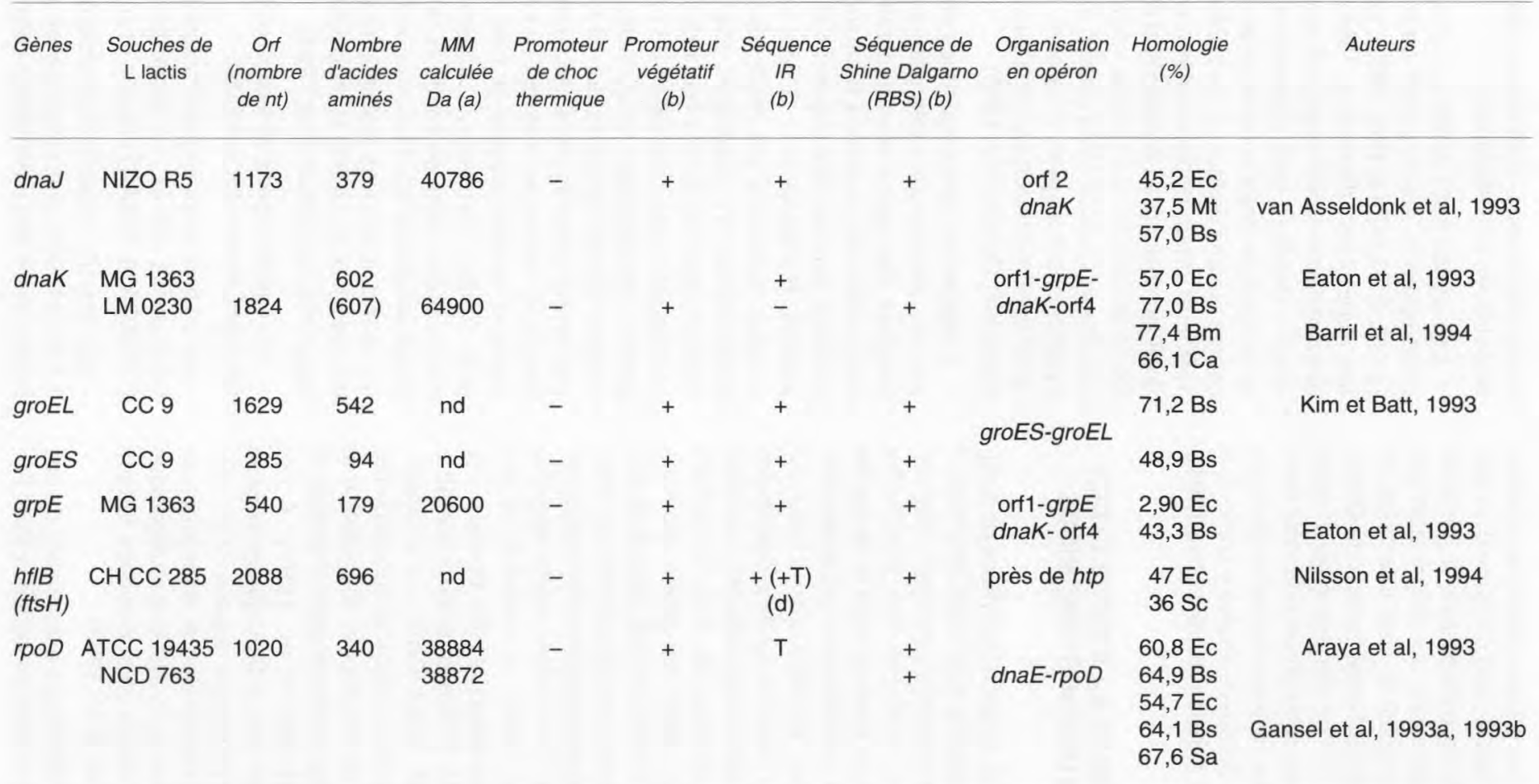

(a) nd : non déterminé par les auteurs ; (b) voir tableau IV ; (c) Bm : Bacillus megaterium ; Bs : Bacillus subtilis ; Ca : Clostridium acetobutylicum ; Ec : Escherichia coli ; Mt : Mycobacterium tuberculosis ; Sc : Saccharomyces cerevisiae; Sa : Staphylococcus aureus ; (d) T : terminateur

(a) nd: not determined by the authors; (b) see table IV; (c) Bm: Bacillus megaterium; Bs: Bacillus subtilis; Ca: Clostridium acetobutylicum; Ec: Escherichia coli; Mt: Mycobacterium tuberculosis; Sc: Saccharomyces cerevisiae; (d) T: terminator. 
Tableau IV. Séquences nucléotidiques remarquables et conservées chez les gènes de choc thermique de Lactococcus lactis. Remarkable and conserved nucleotidic sequences of the heat shock genes of Lactococcus lactis.

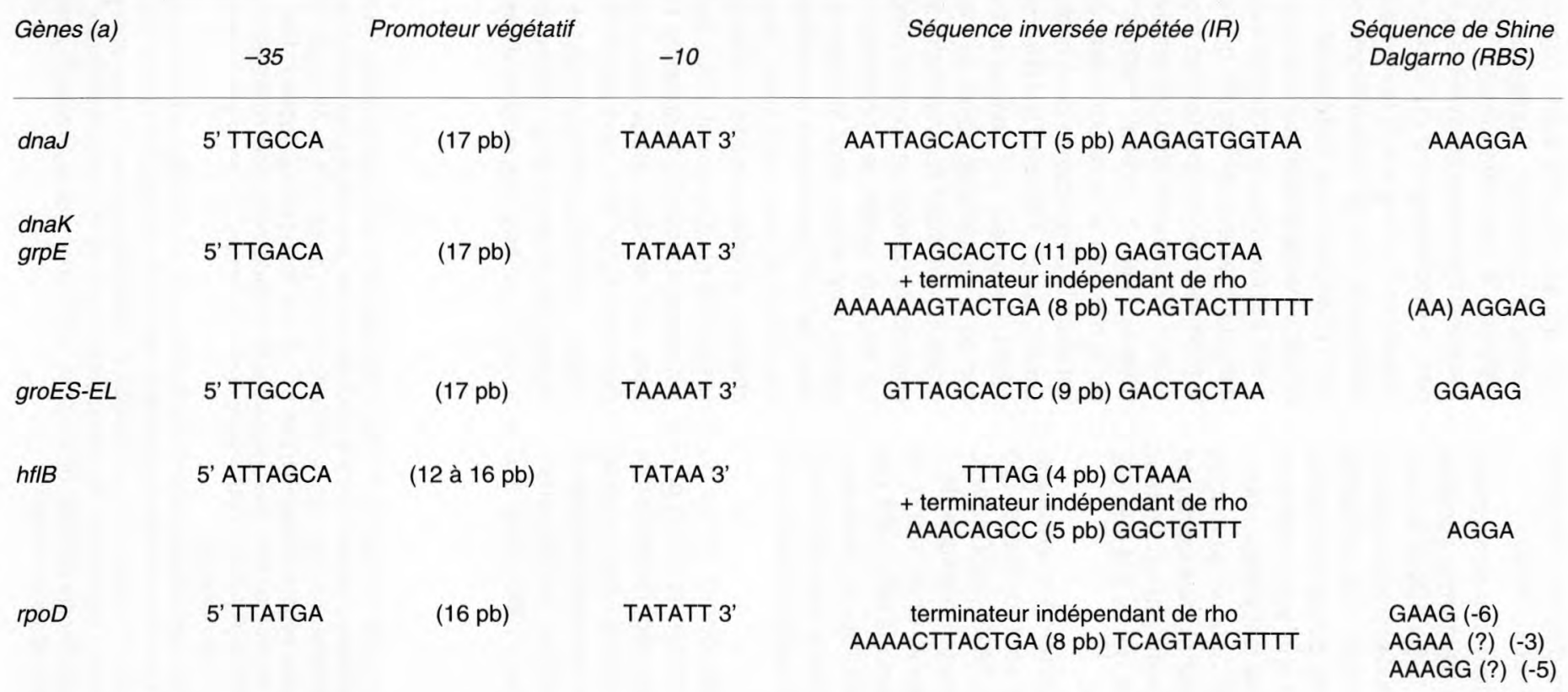

(a) Les séquences se rapportent aux gènes décrits dans le tableau III, isolés à partir des mêmes souches de Lactococcus lactis.

(a) Nucleotidic sequences are those of heat shock genes described in table III, obtained from the same strains of Lactococcus lactis. 
chez $B$ subtilis et chez d'autres bactéries Gram-positives (Eaton et al, 1993) ;

- par un site de fixation des ribosomes, (séquence de Shine Dalgarno) complémentaire de l'ARN 16S (AGGAG pour dnaK) ; chez certains d'entre eux (rpoD) sont inclus une séquence TGN, présente dans environ $50 \%$ des gènes des bactéries Gram-positives et une autre répétition inversée faisant office de terminateur potentiel (Araya et al, 1993) ; aucun ne montre le motif consensuel qui signe l'existence d'un promoteur reconnu par un facteur sigma alternatif, comme $\sigma^{32}$ ou $\sigma^{24}(\sigma E)$ d' $E$ coli (Lindquist, 1986) ;

- parmi une soixantaine de marqueurs, quatre de ces gènes sont localisés, (Le Bourgeois et al, 1992, 1993) (rpoD, dnaK, dnaJ et $g r p E$ ) sur la carte génétique de $L$ lactis 1403 (Le Bourgeois et al, 1993), et de $L$ lactis subsp cremoris MG 1363 (Le Bourgeois et al, 1995). Sur la première, dnaJ flanque $r p o D$; sur la seconde dnaJ, $r p o D$ et dnak divisent le chromosome en trois parties égales.

Le séquençage des gènes isolés ou groupés en opérons et de leurs zones promotrices exclusivement végétatives remet en question l'universalité du seul modèle accepté ces dernières années (Neidhardt et al, 1984 ; Neidhardt et VanBogelen, 1987 ; Lindquist et Craig, 1988), celui d' $E$ coli, qui se résume à une commutation transcriptionnelle. Classiquement, la réponse à une stimulation thermique s'accompagne dans les minutes qui suivent par le remplacement du facteur sigma majeur $\left(\sigma^{70}\right)$, dégradé à température non permissive, par le facteur sigma alternatif $\left(\sigma^{32}\right)$ (Calendar et al, 1988) stable et même activé à haute température et dont la synthèse est par ailleurs amplifiée sous l'effet d'un autre facteur ( $\sigma^{24} \mathrm{ou} \sigma^{\mathrm{E}}$ ) qui reconnaît le promoteur P3 de rpoH et son propre promoteur $\mathrm{P} 2$, assurant ainsi son autorégulation (Bukau, 1993; Yura et al, 1993). L'inflexion de la réponse et le retour à état de repos est sous la dépendance de $4 \mathrm{hsps}$, DnaK, GroEL, GrpE, HflB ou FtsH (Herman et al, 1995). Le facteur $\sigma^{32}$ conduit la transcription de la vingtaine de gènes qu'il reconnaît grâce à leur motif hs (heat-shock) et qui composent ce régulon conventionnel (fig 2). La majeure partie des protéines dont les gènes sont inclus dans le régulon dépendant de $\sigma^{32}$ présentent une affinité pour d'autres protéines cellulaires, cibles de la chaleur, qu'elles assistent, désagrègent, restaurent ou dégradent. Elles participent vraisemblablement à la protection des cellules ou à leur sauvetage lorsqu'elles sont menacées par leur environnement thermique. Le facteur $\sigma^{24}$ ou $\sigma^{\mathrm{E}}$ classé dans le groupe des facteurs ECF (extracytoplasmic function) contrôle l'expression d'une dizaine

Fig 2. Régulation de l'expression des gènes de choc thermique chez Escherichia coli et Bacillus subtilis. A. Systèmes multigéniques impliqués dans la réponse au choc thermique chez ces 2 espèces. B. Modes de régulation de l'expression des gènes. $\sigma^{70}(r p o D) ; \sigma^{32}(r p o H) ; \sigma^{24}$ ou $\sigma^{\mathrm{E}}(r p o E) ; \mathrm{P}: \mathrm{ARN-}$ polymérase; PV : promoteur végétatif dépendant de $\sigma^{70}\left(E\right.$ coli) ou de $\sigma^{43}$ (B subtilis) ; Phs : promoteur de choc thermique reconnu par $\sigma^{32}$; hse : séquence IR (inversion répétée) constituant l'élément de choc thermique (CIRCE) de $B$ subtilis; ORF : cadre ouvert de lecture ; $T$ : terminateur potentiel (IR) ; $\mathrm{R}$ : protéine «répresseur» hypothétique.

Regulation of the heat shock genes expression in Escherichia coli and Bacillus subtilis. A. Multigenic systems implicated in the heat shock response in these 2 bacterial species. B. Ways of regulation of gene expression. $\sigma^{70}(\mathrm{rpoD}) ; \sigma^{32}(\mathrm{rpoH}) ; \sigma^{24}$ ou $\sigma^{E}$ (rpoE); P: RNA-polymerase; Pv: vegetative promotor $\sigma^{70}\left(\mathrm{E}\right.$ coli) or $\sigma^{43}$ (B subtilis) dependent; Phs: heat shock promotor $\sigma^{32}$ dependent; hse: inverted repeat (IR) or heat shock element (CIRCE) of B subtilis; ORF: open reading frame; T: potential terminator (IR); R: hypothetic repressor protein 


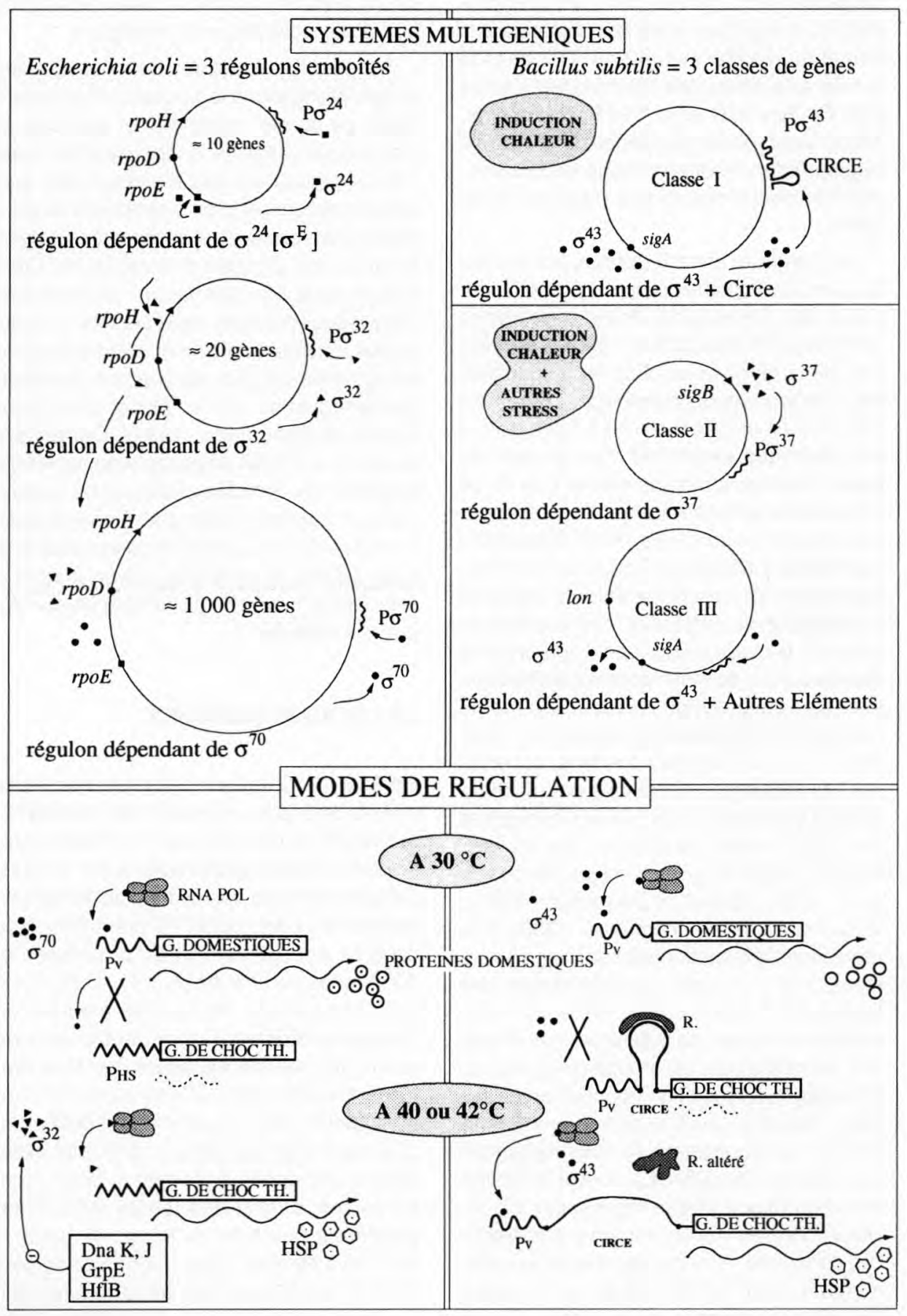


de gènes (dont rpoHet rpoE) dont les produits sont impliqués dans la croissance aux températures élevées $\left(42-44^{\circ} \mathrm{C}\right)$; dans la survie aux épreuves thermiques létales $\left(50^{\circ} \mathrm{C}\right.$ ) (Rouvière et al, 1995 ; Raina et al, 1995). Les produits des gènes de ce second régulon interviendraient dans le compartiment extracytoplasmique (Mecsas et al, 1993).

Au contraire chez B subtilis, les promoteurs hs sont absents ; ceux d'E colifusionnés à des gènes rapporteurs sont inactifs (Wetzstein et Schumann, 1990). L'expression de la réponse au choc thermique procède de promoteurs canoniques, reconnus par $\sigma^{43}$ (homologue de $\sigma^{70} d^{\prime} E$ coli), qui ont été identifiés en amont d'un groupe de gènes thermorégulés de classe I, et d'une séquence inversée répétée de $9 \mathrm{pb}$ appelée CIRCE (controlling IR of chaperone expression) (Zuber et Schumann, 1994), habituellement intercalée entre le signal de transcription et le début du cadre ouvert de lecture. La fixation supposée d'une protéine répresseur sur ce motif récurrent limiterait ou interdirait l'expression du gène. Ce même répresseur constituerait le capteur ou "senseur", l'une des cibles sensibles, qui obéirait à la sollicitation thermique par un changement conformationnel, lequel provoquerait son détachement de l'IR suivi par la transcription du gène correspondant (Wetzstein et al, 1992 ; Zuber et Schumann 1994 ; Völker et al, 1994 ; Yura et al, 1993). À la régulation transcriptionnelle positive s'exprimant, chez E coli, par une permutation des facteurs $\sigma^{70}$ aux facteurs $\sigma^{32}$ et $\sigma^{24}$ euxmêmes emboîtés, se substitue chez $B$ subtilis, au moins pour un groupe de gènes, un mécanisme à régulation négative en cis faisant intervenir, outre le couple promoteurfacteur sigma végétatif, la reconnaissance d'un élément hs (CIRCE), couplé à l'altération d'un hypothétique répresseur (fig 2). Deux modèles nous sont ainsi proposés : l'un complexe, étranger semble-t-il aux éléments aujourd'hui reconnus, par l'analyse des gènes séquencés, chez $L$ lactis ; l'autre moins élaboré, qui traduit une riposte plus fruste aux sollicitations thermiques.

Ne possédant que des données éparses et des informations lacunaires, nous retiendrons ce dernier modèle pour expliciter le mécanisme global de la réponse chez $L$ lactis. La présence des IR ayant été, par ailleurs, reconnue chez une dizaine de bactéries Gram positives et apparentées, appartenant à des groupes très variés, de Clostridium sp à Mycobacterium tuberculosis, chez lesquelles elles représentent le motif le plus fortement conservé, il apparaît qu'un tel système est plus ancien que le mécanisme régulateur mis en place, chez E coli (Zuber et Schumann, 1994). Cependant Duwat et al (1995) proposent une autre voie originale de contrôle impliquant à la fois, par une réaction en cascade, Rec A et HfIB. L'intervention de cette dernière protéine sous-entend la médiation par un facteur $\sigma$ alternatif du type $\sigma^{32}$, qui, jusqu'à ce jour, n'a pas été identifié.

\section{LA THERMOTOLÉRANCE}

De 30 à $43^{\circ} \mathrm{C}$, le taux de croissance d'une culture de $L$ lactis varie peu. De 40 à $48^{\circ} \mathrm{C}$, la viabilité d'une population cellulaire prélevée en phase exponentielle (à $30^{\circ} \mathrm{C}$ ) n'est pas affectée; elle décroit trés rapidement en revanche à partir de $50^{\circ} \mathrm{C}$ pour n'être que de $8 \%$ et $0,06 \%$ après 15 minutes et 30 minutes de chauffage à $51^{\circ} \mathrm{C}$ (fig 3 ). A $55^{\circ} \mathrm{C}$ et au-delà, les cellules meurent en quelques minutes. Cependant le taux de survie des cellules exposées aux températures élevées dépend des conditions de croissance. Ainsi, il est considérablement plus élevé si les bactéries, avant d'être exposées à une épreuve thermique létale, sont soumises, durant des temps qui correspondent à peu près au temps de génération (30 à $40 \mathrm{~min}$ ) à des températures de 5 à $10^{\circ} \mathrm{C}$ supérieures à la température habituelle de croissance. L'appréciation quan- 

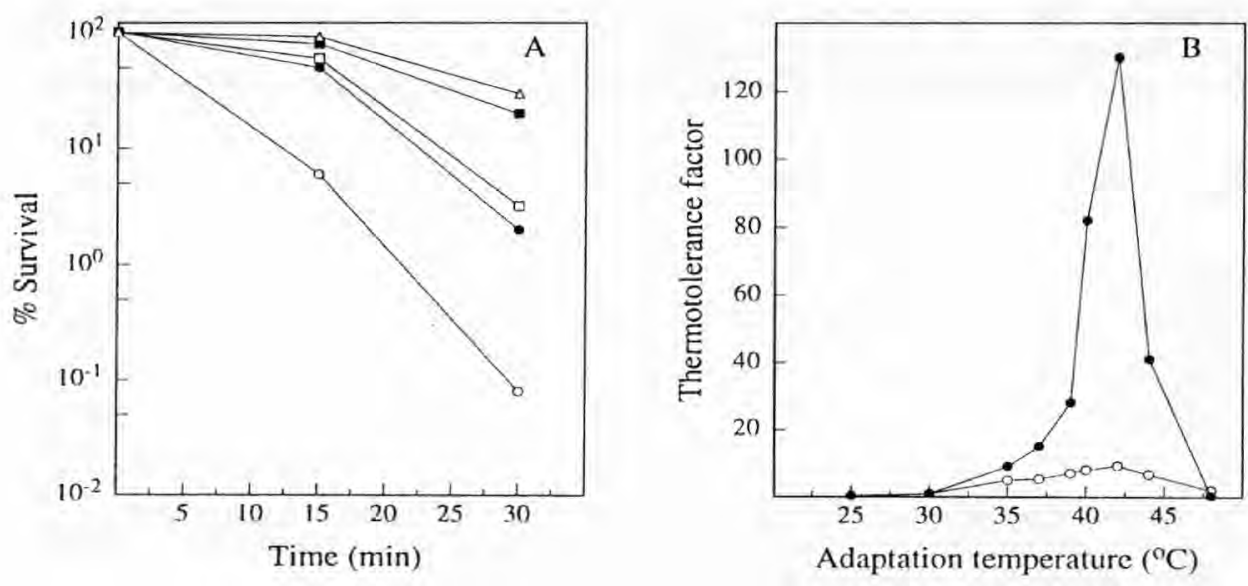

Fig 3. A. Thermotolérance (à $52^{\circ} \mathrm{C}$ ) développée par diverses cultures de Lactococcus lactis subsp lactis IL 1403 à $30^{\circ} \mathrm{C}(\mathrm{O}), 35^{\circ} \mathrm{C}(\bullet), 37^{\circ} \mathrm{C}(\square), 40^{\circ} \mathrm{C}(\mathbf{\square}), 42^{\circ} \mathrm{C}(\Delta)$. B. Facteurs de thermotolérance (à $52^{\circ} \mathrm{C}$ ) après $15 \mathrm{~min}(\mathrm{O})$ ou $30 \mathrm{~min}(\bullet)$ développés par diverses cultures de L lactis subsp lactis IL 1403. Le facteur de thermotolérance est égal au rapport du pourcentage de survie d'une population "conditionnée" ou adaptée à différentes températures (de 25 à $48^{\circ} \mathrm{C}$ ) exposée à $52^{\circ} \mathrm{C}$ pendant $30 \mathrm{~min}$, sur le pourcentage de survie de la population témoin cultivée à $30^{\circ} \mathrm{C}$ (d'après Boutibonnes et al, 1995).

A. Thermotolerance (at $52^{\circ} \mathrm{C}$ ) developed by Lactococcus lactis subsp lactis $I \mathrm{~L} 1403$ at $30^{\circ} \mathrm{C}(\mathrm{O}), 35^{\circ} \mathrm{C}$ (•), $37^{\circ} \mathrm{C}(⿻), 40^{\circ} \mathrm{C}(\mathbf{\Delta}), 42^{\circ} \mathrm{C}(\Delta)$. B. Thermotolerance factors $\left(\right.$ at $52^{\circ} \mathrm{C}$ ) after $15 \mathrm{~min}(\mathrm{O})$ or $30 \mathrm{~min}$ (-) obtained with growing cells of $\mathrm{L}$ lactis subsp lactis IL 1403. The enhanced thermal resistance was expressed as "thermotolerance factor", ie the ratio of per cent survival of bacterial cells grown at different temperatures (from 35 to $42^{\circ} \mathrm{C}$ ) to per cent survival of control cells grown at $30^{\circ} \mathrm{C}$; at this temperature the thermotolerance factor is equal to 1 (according to Boutibonnes et al, 1995).

titative de ce phénomène d'exhaussement de la survie cellulaire basale, appelé résistance induite ou thermotolérance, observé chez tous les représentants du monde vivant (bactéries, champignons, plantes et animaux) (Lindquist et Craig, 1988) est fournie par le facteur de thermotolérance, c'està-dire le rapport du pourcentage de survie d'une population adaptée ou conditionnée à telle épreuve sur le pourcentage de survie d'une population témoin, «naïve» cultivée à $30^{\circ} \mathrm{C}$ (Boutibonnes et al, 1991). Ce facteur covarie avec la température de préculture : égal à 1 à $30{ }^{\circ} \mathrm{C}$, il est maximal à $42^{\circ} \mathrm{C}$ où sa valeur dépasse 100 (fig 3 ), puis il décroît très vite quand la température d'adaptation se rapproche de la température d'épreuve $\left(52^{\circ} \mathrm{C}\right)$. Si les cellules "conditionnées" par la chaleur, qui antici- pent ou se préparent à une épreuve thermique létale, meurent lentement, le traitement d'adaptation entraîne de surcroît une tolérance hétérologue vis-à-vis d'autres modalités qui menacent la survie cellulaire ; ces résistances croisées s'expriment vis-àvis d'agents létaux, dont la nature et les fonctions cytotoxiques sont éloignées de celles de la chaleur (tableau V). Ainsi un prétraitement à $42{ }^{\circ} \mathrm{C}$ élève considérablement la résistance «constitutive» des bactéries à l'alcool et au $\mathrm{pH}$ acide (on parle d'alcoolotolérance ou de $\mathrm{pH}$ - tolérance). II n'a qu'un effet discret sur la résistance à l'eau oxygénée, au sel et aux $U V_{254} \mathrm{~nm}$. Le traitement préalable par les températures modérées a également pour conséquence d'inhiber la lyse cellulaire des bactéries lysogènes (ici $L$ lactis $A 15$, abritant le phage 
Tableau V. Tolérances croisées exprimées par une culture à $42^{\circ} \mathrm{C}$ de Lactococcus lactis subsp lactis IL 1403 vis-à-vis de diverses épreuves létales.

Cross tolerances expressed by growing cells of Lactococcus lactis against some lethal challenges.

$\begin{array}{lcccccc}\text { Agents } & \text { Chaleur } & \text { Alcool } & \mathrm{NaCl} & \mathrm{H}_{2} \mathrm{O}_{2} & \mathrm{UV}_{254 \mathrm{~nm}} & \mathrm{pH} \\ \begin{array}{l}\text { Valeur de } \\ \text { l'épreuve }\end{array} & 52^{\circ} \mathrm{C} & 20 \%(\mathrm{~V} / \mathrm{V}) & 3,5 \mathrm{~mol} / \mathrm{l} & 0,05 \% & 100 \mathrm{~J} / \mathrm{m}^{2} & 4,0 \\ \begin{array}{l}\text { Facteur de } \\ \text { tolérance (a) }\end{array} & 130 & 100 & 10 & 7 & 4,5 & 129\end{array}$

(a) Le facteur de tolérance à une épreuve donnée est le rapport de la survie d'une population cellulaire conditionnée par un prétraitement à $42^{\circ} \mathrm{C}$ durant $30 \mathrm{~min}$, sur la survie d'une population témoin cultivée dans les mêmes conditions à $30^{\circ} \mathrm{C}$ et soumise à cette même épreuve.

(a) The tolerance factor toward a particular lethal challenge is the ratio of per cent survival of bacterial cell adapted to $42{ }^{\circ} \mathrm{C}$ during $30 \mathrm{~min}$ to per cent survival of control cells grown at $30^{\circ} \mathrm{C}$, exposed to the same challenge.

A15) provoquée par l'induction expérimentale du prophage (Boutibonnes et al, 1991): le choc thermique (de 30 à $40^{\circ} \mathrm{C}$ ) est d'autant plus efficace qu'il est plus précoce, c'est-à-dire plus proche de l'administration de l'agent SOS-inducteur qui déclenche la lyse (mitomycine C ou UV254 nm).

La synthèse d'hsps, mais aussi le développement de la thermotolérance sont conditionnées aux traitements des cellules par diverses formes de stress, (tableau II) lesquels dans la majorité des cas entraînent des dommages protéiques (Hightower, 1991). Toutefois, une question reste toujours posée : elle concerne la relation hypothétique qui lierait, sans réserve, la néosynthèse d'une ou de quelques hsps à la capacité nouvellement acquise par les cellules d'outrepasser l'épreuve létale (Parsell et Lindquist, 1994). De nombreux travaux, portant sur les bactéries mais aussi sur les cellules eucaryotes en culture in vitro, étayent ou infirment l'existence de cette interdépendance ; par exemple chez E coli, la surexpression des gènes du régulon $\sigma^{32}$, obtenue en plaçant le gène $\mathrm{rpoH}$ sous la commande d'un promoteur tac inductible par I'IPTG (isopropyl thio $\beta$-galactoside), suivi de la synthèse d'hsps à hauts niveaux endocellulaires, ne conduit pas au développement de thermotolérance (VanBogelen et al, 1987). Chez la levure, la thermotolérance peut se développer en l'absence de l'hsp majeure ou hsp 104 identifiée par Sanchez et Lindquest (1990), et de toute néosynthèse protéique... (Sanchez et Lindquist, 1990) et de toute néosynthèse protéique (De Virgilio et al, 1991).

Ainsi chez $L$ lactis des thermomimétiques qui induisent la synthèse des hsps, impliquées dans le surcroît de résistance à la chaleur (DnaK, GroEL, GrpE, hsp 104 ou $\mathrm{ClpB})$ n'affectent pas la résistance thermique naturelle des cellules : c'est le cas de la plupart des antibiotiques. Inversement, l'acquisition de l'aptitude nouvelle à tolérer l'épreuve thermique létale, acquise après traitement par le chlorure mercurique et l'azide de sodium ou consécutive à l'entrée des cellules en phase stationnaire est découplée de la capacité de surexprimer les hsps. Soulignons enfin, que chez $L$ lactis, à l'inverse de ce qui se passe chez $E$ coli, des agents réputés pour déclencher 
chez des cellules eucaryotes en culture la synthèse des hsps et/ou la thermotolérance, sont sans effet.

Bien que leur présence soit nécessaire, la responsabilité des hsps dans la thermotolérance est sans cesse remise en question par des résultats expérimentaux contradictoires. II y a vraisemblablement trois explications à cette incertitude : 1) les hsps naturellement présentes, quelquefois même abondantes, ne nécessiteraient qu'une "activation" par les traitements de conditionnement pour devenir fonctionnelles et intervenir dans les processus de sauvetage thermique. 2) Les hsps "classiques" ne seraient peut-être pas les seuls agents prévenant ou restaurant les dégâts protéiques, impliqués dans les tolérances (Parsell et Lindquist, 1994). D'autres facteurs de protection pourraient être associés à l'induction de systèmes multigéniques discrets (tel le régulon $\sigma^{24}$ d'E coli dont nous méconnaissons la fonction exacte). 3) L'hyperproduction ou l'activation des hsps, par mise en conformité des chaînes peptidiques, augmente la capacité de protéolyse endocellulaire. Certaines hsps qui sont aussi des protéases, exercent leur activité sur d'autres hsps les faisant “disparaître" après qu'elles ont assuré leur office de restauration : leur trace même ne serait pas repérable par analyse électrophorétique.

\section{CONCLUSION}

Si depuis 5 ans environ l'on s'achemine vers la connaissance plus précise des termes (inducteurs ou stresseurs, cibles ou senseurs, protéines, gènes) qui, chez $L$ lactis, constituent la phénoménologie de la réponse au choc thermique, il reste encore beaucoup à faire pour comprendre le déclenchement et la cascade des événements qui aboutissent à ce phénomène "chaotique", obéissant à d'imperceptibles fluctuations des conditions initiales (ici une augmentation de quelques ${ }^{\circ} \mathrm{C}$ ). Demeurent notamment ces trois questions : Quel est le mode de régulation de l'expression des gènes de choc thermique? Thermotolérance et hsps sont-elles en étroite connexion? Si c'est le cas, comment se réalisent, surveillance, prévention, mais aussi désagrégation et restauration de l'arsenal protéique immature ou endommagé par la machinerie de chaperonnage?

La problématique des stress, c'est-àdire la prise en compte de l'environnement et de ses paramètres, qui forment avec I'hôte des niches un couple insécable, s'est doublement élargie. À l'instar du choc thermique, les fluctuations de $\mathrm{pH}$ (acide ou alcalin), d'osmolarité, d'oxygénation, l'abaissement de la temperature $\left(4^{\circ} \mathrm{C}\right.$ ) (Panoff et al, 1994), la pénurie en nutriment (par exemple l'épuisement de la source de carbone: Hartke et al, 1994) déclenchent chez les bactéries lactiques mésophiles -et comme on s'y attendait-, une réponse duelle, couplant la synthèse de nouvelles protéines de stress à des états de tolérance, ou de multitolérance, quelquefois élevée.

La réponse au choc thermique, qui semble constituer une réserve de mémoire déposée dans le génome sous forme de traces mnésiques, de projets prêts à être réactualisés en certaines occasions, est maintenant reconnue chez d'autres bactéries lactiques senso lato atypiques : Enterococcus faecalis, dont l'un des traits singuliers est une résistance peu ordinaire à de nombreuses conditions "extrêmes" (Boutibonnes et al, 1993) ; Streptococcus thermophilus dont la synthèse d'hsps, stimulée à haute température $\left(52^{\circ} \mathrm{C}\right)$, est accompagnée d'une viabilité importante à $58^{\circ} \mathrm{C}$ (Auffray et al, 1995).

Le phénomène s'impose comme le modèle d'étude de la régulation de l'expression de systèmes multigéniques conduisant à une authentique différenciation métabolique : la cellule stressée n'est plus tout à 
fait la même que la cellule «naïve». Les forces sélectives, mais aussi les contraintes endogènes (génétiques ou "mécaniques") ont agi pour maintenir au sein de la cellule (ou des organismes) ce que les processus évolutifs avaient érigé afin que cette cellule (ou ces organismes) échappent aux conditions adverses qui menaçaient à la fois l'intégrité de l'une (ou des autres) et la descendance de l'espèce. II n'y a pas, dans ce type de réponse, de commune mesure entre l'ampleur des conséquences due à une véritable commotion métabolique (le plus souvent bénéfique) et l'insignifiance des causes (quelques degrés thermiques). Ce qui paraît être une riposte désordonnée et disproportionnée, n'est pas, semble t-il, un défaut ou une crise de l'ordre biologique habituel ; elle n'est qu'une des conditions qui a autorisé l'existence et la permanence de cet ordre. C'est parce que, par essence, le désordre est versatile, qu'il se renouvelle sans cesse pour se convertir en un autre désordre jusqu'à paraître incernable, qu'une relation intelligible entre une cause et sa conséquence peut s'installer et légiférer de manière immuable la fonction des systèmes biologiques.

\section{REMERCIEMENTS}

Je voudrais associer à la rédaction de cet article tous les membres du Laboratoire de microbiologie de l'environnement; leurs critiques et leurs suggestions m'ont été précieuses.

Je remercie particulièrement pour leur aide, $B$ Thammavongs qui a relu et corrigé le manuscrit ; A Hartke qui m'a fourni les électrophorégrammes; J Frère qui a réalisé la mise en page du schéma de régulation.

Mes plus vifs remerciements vont également à $\mathrm{M}$ Lautier et $\mathrm{P}$ Ritzenthaler (Université de Toulouse), à P Duwat (Inra, Jouy-en-Josas) qui m'ont transmis quelques documents indispensables dont certains étaient inédits. Je remercie $M$ Lamare qui a assuré la frappe du texte et la composition des tableaux.

\section{RÉFÉRENCES}

Araya T, Ishibashi N, Shimamura S, Tanaka K, Takahashi $H$ (1993) Genetic and molecular analysis of the rpoD gene from Lactococcus lactis. Biosci Biotechnol Biochem 57, 88-92

Arnosti DN, Singer VL, Chamberlin MJ (1986) Characterization of heat shock in Bacillus subtilis. $J$ Bacteriol 168, 1243-1249

Auffray $Y$, Gansel X, Thammavongs B, Boutibonnes P (1992) Heat shock-induced protein synthesis in Lactococcus lactis subsp lactis. Curr Microbiol 24, 281 284

Auffray Y, Lecesne E, Hartke A, Boutibonnes P (1995) Basic features of the Streptococcus thermophilus heat shock response. Curr Microbiol 30, 87-91

Barril J S, Kim S G, Batt C A (1994) Cloning and sequencing of the Lactococcus lactis subsp lactis dnak gene using a PCR - based approach. Gene 142, 91-96

Boutibonnes P, Gillot B, Auffray $Y$, Thammavongs B (1991) Heat shock induces thermotolerance and inhibition of lysis in a lysogenic strain of Lactococcus lactis. Int J Food Microbiol 14, 1-10

Boutibonnes $\mathrm{P}$, Tranchard $\mathrm{C}$, Hartke A, Thammavongs $B$, Auffray $Y$ (1992) is thermotolerance correlated to heat shock protein synthesis in Lactococcus lactis subsp lactis? Int J Food Microbiol 16, 227-230

Boutibonnes P, Giard JC, Hartke A, Thammavongs B, Auffray $Y$ (1993) Characterization of the heat shock response in Enterococcus faecalis. Antonie van Leeuwenhoek 64, 47-55

Boutibonnes P, Bisson V, Thammavongs B, Hartke A, Panoff JM, Benachour A, Auffray Y (1995) Induction of thermotolerance by chemical agents in Lactococ. cus lactis IL 1403. Int J Food Microbiol 25, 83-94

Brenner DJ (1984) Enterobacteriaceae. In: Bergey's Manual of Determinative Bacteriology (Murray RGE, Brenner DJ, Bryant MP, Holt JG, Krieg NR, Moulder JW, Pfennig N, Sneath PHA, Staley JT, eds) The Williams and Wilkins Company, Baltimore

Burton Z, Burgess RR, Lin J, Moore D, Holder S, Gross CA (1981) The nucleotide sequence of the cloned $r p o D$ gene for the RNA polymerase sigma subunit from Escherichia coli K 12. Nucleic Acids Res 9, 2889-2903

Bukau B (1993) Regulation of the Escherichia coli heat shock response. Mol Microbio/ 9, 671-680

Calendar R, Erickson JW, Halling C, Nolte A (1988) Deletion and insertion mutations in the rpoH gene of Escherichia coli that produce functional $\sigma^{32}$. $J \mathrm{Bac}$ teriol 170, 3479-3484

Craig EA, Gambill BD, Nelson RJ (1993) Heat shock proteins: molecular chaperones of protein biogenesis. Microbiol Rev 57, 402-414

De Virgilio C, Piper P, Boller T, Wienken A (1991) Acquisition of thermotolerance in Saccharomyces cerevi- 
siae without heat shock protein hsp 104 and in the absence of protein synthesis. FEBS Lett $288,86-90$

Duwat $P$, Ehrlich SD, Gruss A (1995) The recA gene of Lactococcus lactis: Characterization and involvement in oxydative and thermal stress. Mol Microbiol $17,1121-1131$

Eaton T, Shearman C, Gasson M (1993) Cloning and sequence analysis of the dnaK gene region of Lactococcus lactis subsp lactis. I Gen Microbiol 139, 3253-3254

Gansel X, Dutreix M, Hartke A, Boutibonnes P, Auffray $Y$ (1993a) Partial characterization of an rpoD - like gene of Lactococcus lactis subsp lactis ML3 with a polymerase chain reaction based approach. Curr Microbiol 27, 267-271

Gansel X, Hartke A, Boutibonnes P. Auffray Y (1993b) Nucleotide sequence of the Lactococcus lactis NCDO 763 (ML3) rpoD gene. Biochim Biophys Acta 1216, 115-118

Georgopoulos C, Ang D, Liberek K, Zylicz M (1990) Properties of the Escherichia coli heat shock proteins and their role in bacteriophage growth. In: Stress proteins in biology and medicine (Morimoto RI, Tissière A, Georgopoulos C, eds). Cold Spring Harbor Laboratory Press, New York

Hartke A, Bouche S, Gansel X, Boutibonnes P, Auffray $Y(1994)$ Starvation-induced stress resistance in Lactococcus lactis subsp lactis IL 1403. Appl Environ Microbiol 60, 3474-3478

Herman C, Thevenet D, D'Ari R, Bouloc P (1995) Degradation of $\sigma^{32}$, the heat shock regulation in Escherichia coli is governed by HflB. Proc Natl Acad Sci USA 92, 3516-3520

Hightower LE (1991) Heat shock, stress proteins, chaperones and proteotoxicity. Cell 66, 191-197

Kim SG, Batt CA (1993) Cloning and sequencing of the Lactococcus lactis subsp lactis GroESL operon. Gene 127, 121-126

Le Bourgeois P, Mata M, Ritzenthaler P (1989) Genome comparison of Lactococcus strains by pulse-field gel electrophoresis. FEMS Microbiol Lett 59, 6570

Le Bourgeois P. Lautier M, Mata M. Ritzenthaler P (1992) Physical and genetic map of the chromosome of Lactococcus lactis subsp lactis IL 1403. J Bacteriol $174,6752-6762$

Le Bourgeois P, Lautier M, Ritzenthaler P (1993) Chromosome mapping in lactic acid bacteria. FEMS Microbiol Rev 12, 109-124

Le Bourgeois P, Lautier M, Van Den Berghe L, Gasson MJ, Ritzenthaler P (1995) Physical and genetic map of the Lactococcus lactis subsp cremoris MG 1363 chromosome: Comparison with that of Lactococcus lactis subsp lactis IL 1403 reveals a large genome inversion. J Bacteriol 177, 2840-2850

Lemaux PG, Herendeen SL, Bloch PL, Neidhardt FC (1978) Transient rate of synthesis of individual poly- peptides in Escherichia coli following temperature shifts. Cell 13, 427-434

Lindquist SL (1986) The heat shock response. Annu Rev Biochem 55, 1151-1191

Lindquist SL, Craig EA (1988) The heat shock proteins. Annu Rev Genet 26, 631-677

Mecsas J, Rouvière PE, Erickson JW, Donohue TJ, Gross CA (1993) The activity of $\sigma^{E}$ an Escherichia coli heat inducible $\sigma$ factor is modulated by expression of outer membrane proteins. Genes Dev 7. 2619-2628

Morimoto RI, Tissières A, Georgopoulos C (1994) Progress and perpectives in the biology of heat shock proteins and molecular chaperones. In: The biology of heat shock proteins and molecular chaperones (Morimoto RI, Tissières A, Georgopoulos C, eds) Cold Spring Harbor Laboratory Press, New York

Neidhardt FC, VanBogelen RA, Vaugh N (1984) The genetics and regulation of heat shock proteins. Annu Rev Genet 18, 295-329

Neidhardt FC، VanBogelen RA (1987) Heat shock response. In: Escherichia coli and Salmonella typhimurium: cellular and molecular biology (Ingraham JL. Low KB, Magasanik B. Schaechter M. Umbarger $H E$, eds) Library of Congress, Washington DC

Nilsson D, Lauridsen AA, Tomoyasu T, Ogura T (1994) A Lactococcus lactis gene encodes a membrane protein with putative ATP-ase activity that is homologous to the essential Escherichia coli fts $\mathrm{H}$ gene product. Microbiology 140, 2601-2610

Panoff JM, Legrand S, Thammavongs B, Boutibonnes P (1994) The cold shock response in Lactococcus lactis subsp lactis. Curr Microbiol 29, 213-216

Parsell DA, Lindquist SL (1994) Heat shock proteins and stress tolerance. In: The biology of heat shock proteins and molecular chaperones (Morimoto RI. Tissières A, Georgopoulos C, eds) Cold Spring Harbor Laboratory, New York

Raina S, Missiakas D, Georgopoulos C (1995) The rpoE gene encoding the $\sigma^{E}\left(\sigma^{24}\right)$ heat shock sigma factor of Escherichia coli. EMBO J 14, 1043-1055

Ritossa F (1962) A new puffing pattern induced by temperature shock and DNP in Drosophila. Experientia $18,571-573$

Rouvière PE, De Las Penas A, Mecsas J, Lu Z, Rudd $\mathrm{KE}$, Gross CA (1995) rpoE, the gene encoding the second heat shock sigma factor, $\sigma^{E}$ in Escherichia coli. EMBO J 14, 1032-1042

Sanchez Y, Lindquist S (1990) HSP 104 required for induced thermotolerance. Science 248, 1112-1115

Sandine WE, Radich PC, Elliker PR (1972) Ecology of the lactic streptococci: a review. J Milk Food Technol $35,176-185$

Tanskanen EI, Tulloch DL, Hillier AJ, Davidson BE (1990) Pulsed-field gel electrophoresis of Sma I digest of 
lactococcal genomic DNA, a novel method of strain identification. Appl Environ Microbiol 56, 3105-3111

Tissières A, Mitchel HK, Tracy VM (1974) Protein synthesis in salivary glands of Drosophila melanogaster. relation to chromosome puffs. J Mol Biol 84, 389398

Van Asseldonk M, Simons A, Visser $H$, de Vos WM, Simons G (1993) Cloning, nucleotide sequence and regulatory analysis of the Lactococcus lactis dnaJ gene. J Bacteriol 175, 1637-1644

VanBogelen RA, Acton MA, Neidhardt FC (1987) Induction of the heat shock regulon does not produce thermotolerance in Escherichia coli. Gene Dev 1, 525531

Völker U, Engelmann S, Maul B, Riethdorf S, Volker A, Schmid R, Mach H, Hecker M (1994) Analysis of the induction of general stress proteins of Bacillus subtilis. Microbiology 140, 741-752

Wawrzynow A, Wojtkowiak D, Marszalek J, Banecki B, Jonsen M, Graves B, Georgopoulos C, Zylicz M (1995) The ClpX heat shock protein of $E$ coli the ATP dependent substrate specificity component of the ClpP - ClpX protease is a novel molecular chaperone. EMBO J 14, 1867-1877
Wetzstein M, Schumann W (1990) Nucleotide sequence of a Bacillus subtilis gene homologous to the grpE gene of Escherichia coli located immediately upstream of the dnaK gene. Nucleic Acids Res 18, 1289

Wetzstein M, Völker U, Dedio J, Lobau S, Zuber U, Schiesswohl M, Herget C, Hecker M, Schumann W (1992) Cloning, sequencing and molecular analysis of the dnaK locus from Bacillus subtilis. J Bacteriol $174,3300-3310$

Whitaker RD, Batt CA (1991) Characterization of the heat shock response in Lactococcus lactis subsp lactis. Appl Environ Microbiol 157, 1408-1412

Yamamori T, Ito K, Nakamura Y, Yura T (1978) Transient regulation of protein synthesis in Escherichia coli upon shift-up of growth temperature. J Bacterio/ 134, 1133-1140

Yura T, Nagai H, Mori H (1993) Regulation of the heat shock response in bacteria. Annu Rev Microbiol 47, 321-450

Zuber U, Schumann W (1994) CIRCE, a novel heat shock element involved in regulation of heat shock operon dnaK of Bacillus subtilis. J Bacteriol 176, $1359-1363$ 